\title{
Experimental Design and Power Calculation in Omics Circadian Rhythmicity Detection
}

\author{
Wei Zong ${ }^{1}$, Marianne L. Seney ${ }^{2}$, Kyle D. Ketchesin ${ }^{2}$, Michael T. Gorczyca ${ }^{3}$, Andrew C. \\ Liu $^{4}$, Karyn A. Esser ${ }^{4}$, George C. Tseng ${ }^{1 *}$, Colleen A. McClung ${ }^{2 *}$ and Zhiguang Huo ${ }^{5 *}$ \\ ${ }^{1}$ Department of Biostatistics, University of Pittsburgh, 130 De Soto Street, 15261, PA, USA, ${ }^{2}$ Translational \\ Neuroscience Program, Department of Psychiatry, Center for Neuroscience, University of Pittsburgh, 3811 O'Hara \\ Street, 15213, PA, USA, ${ }^{3}$ Department of Computational and Systems Biology, University of Pittsburgh, 4400 Fifth \\ Ave, 15213, PA, USA, ${ }^{4}$ Department of Physiology and Functional Genomics, University of Florida, 1600 SW Archer \\ Rd, 32603, FL, USA, and ${ }^{5}$ Department of Biostatistics, University of Florida, 2004 Mowry Rd, 32603, FL, USA
}

Received YYYY-MM-DD; Revised YYYY-MM-DD; Accepted YYYY-MM-DD

\begin{abstract}
Circadian clocks are 24-hour endogenous oscillators in physiological and behavioral processes. Though recent transcriptomic studies have been successful in revealing the circadian rhythmicity in gene expression, the power calculation and study design for omics circadian analysis have not been explored. In this paper, we develop a statistical package, namely CircaPower, to perform power calculation for circadian pattern detection. Our theoretical framework is determined by three key factors in circadian gene detection: sample size, intrinsic effect size and sampling design. Via simulations, we systematically investigate the impact of these key factors on circadian power calculation. We demonstrate that CircaPower not only has fast and accurate computing but also is robust against variety of violations of model assumptions. In real applications, we demonstrate the performance of CircaPower using mouse pan-tissue data and human postmortem brain data, and illustrate how to perform circadian power calculation using mouse skeletal muscle microarray pilot data as a case study. Our method CircaPower has been implemented in an $\mathbf{R}$ package, which is made publicly available on GitHub (https://github.com/circaPower/circaPower).
\end{abstract}

\section{INTRODUCTION}

Circadian rhythms are endogenous $\sim 24$ hour oscillations of behavior, physiology, and homeostasis in adaption to the diurnal cycle caused by the earth's daily rotation. The circadian clock is found in virtually all cells throughout the body and controls oscillations in a wide variety of physiological processes, including sleep-wake cycles, body temperature, and melatonin $(2,4,9,19)$. From the literature, the mechanism that drives circadian rhythms is a transcriptionial-translational feedback loop encoded by a set of core clock genes (44), including CLOCK, BMAL1 as the transcriptional activators; and period family (PERl, $P E R 2, P E R 3)$ and cryptochrome family $(C R Y 1, C R Y 2)$ as the major inhibitors. In addition to core clock genes, genomewide transcriptomic studies have revealed additional circadian genes in post-mortem brain $(5,39)$, skeletal muscle (13), liver (17), and blood (31). Human pan-tissue transcriptomic circadian analysis (37) and mouse pan-tissue transcriptomic circadian analysis (49) have shown that the circadian pattern in gene expression could be tissue-specific. Beyond transcriptomic data, circadian rhythmicity was also discovered in other types of omics data including DNA methylation (25), ChIP-Seq (chromatin immunoprecipitation assays with sequencing) (21), proteomics (47), and metabolomics (8). From epidemiology and animal studies, the disruption in clock and circadian gene expression was found to be linked to diseases including type 2 diabetes (42), sleep (31), major depression disorder (23), aging (5), schizophrenia (39), and Alzheimer's disease (24).

Circadian omics studies have become increasingly popular over the years (Figure 1a), because of their capability to decipher circadian rhythms at the molecular level. Though promising, less attention has been paid to the statistical power calculation and sampling design in omics circadian studies. Previous studies have reported the lack of overlapping circadian genes because of smaller number of samples $(15$, 17). This indicates that statistical power, i.e., the probability of successfully detecting the underlying circadian pattern, is not fully considered/justified. In addition to power calculation, the optimal design of collected samples has not been carefully investigated, where the design refers to the distribution of the collected Zeitgeber time (ZT; standardized diurnal time with ZT0 for the beginning of day and ZT12 for the beginning of night). In this paper, we consider two types of sampling

\footnotetext{
*To whom correspondence should be addressed. George C. Tseng, ctseng@pitt.edu; Colleen A. McClung, mcclungca@upmc.edu; Zhiguang Huo, zhuo@ufl.edu 
design: passive or active sampling design. In passive design, investigators have no control of the collected ZT. Such a passive design is commonly seen in studies with human tissues that are difficult to obtain (e.g., post-mortem brain tissues $(5,23,39))$ and the irregular sampling distribution should be considered in power calculation. In contrast, investigators have full control of the sample collection time in an active sampling design. Such an active design is commonly seen in animal studies (49) or human blood studies (31).

For active sampling design, 6 time points (every 4 hours) per cycle across one or multiple full cycles have been widely adopted in many studies (26, 27, 38). Hughes et al. (16) recommended evenly sampling at least 12 time points per cycle (i.e., every 2 hours) across 2 full cycles. For the ease of discussion, we refer to this type of design as the "evenly-spaced sampling design". Though these empirical practices and guidelines were presented and well-received, there were almost no theoretical justifications. Questions remaining include: (i) Is the evenly-spaced sampling design better than irregular (active or passive) sampling design? (ii) More time points in a circadian cycle require more experimental effort and cost. Is 6 or even 12 time points per cycle necessary? (iii) When a pilot dataset is available, how to utilize the circadian effect size information to guide the (active or passive) design of a new study (i.e., the number of samples required to achieve certain pre-set statistical power)?

To fill in these research gaps, we propose a modelbased approach to accurately calculate the circadian power (namely CircaPower), based on a cosinor model $(7,10)$. In the literature, several other computational or non-modelbased algorithms have been developed to detect circadian rhythmicity, including Lomb-Scargle periodograms (12), COSOPT (43), ARSER (48), RAIN (45), and JTK CYCLE (18). These methods assume either mixture of multiple cosinor curves with distinct periods or non-parametric curve fitting to facilitate detection of oscillating transcripts with irregular shape. Although these methods have advantages to detect irregular curves beyond cosinors, the evaluation of power calculation and experimental design using these methods are not feasible since the effect size and data variability are not explicitly defined and modeled $(15,22)$. Moreover, in many human studies with passive sampling design (e.g., post-mortem brain studies in our later applications), the noncosinor irregular curves cannot be accurately estimated and validated due to small sample size and larger heterogeneity in human. Therefore, we build our power calculation framework based on the cosinor model and rigorously evaluate its type I error control, statistical power, and robustness against model assumptions. To the best of our knowledge, this is the first theoretical methodology developed for circadian power calculation in omics data. The unique contribution of this paper includes: (i) identifying factors related to the statistical power of circadian rhythmicity detection, including sample size, intrinsic effect size and sampling design; (ii) developing CircaPower, an analytical solution based on a closed-form formula, for fast and accurate circadian power calculation; (iii) demonstrating via simulations that the evenly-spaced sampling design is superior because of its phase-invariant property, which is also corroborated by theoretical proofs; (iv) illustrating how to calculate statistical power and to design a circadian experiment with pilot data via a case study; (v)

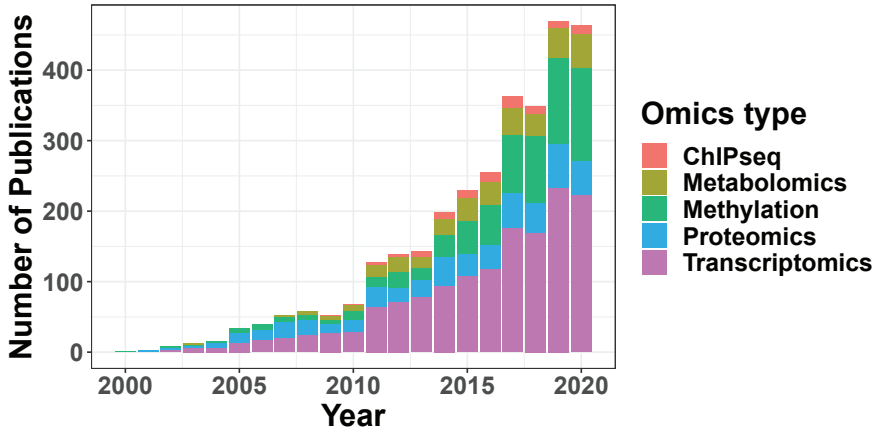

(a)

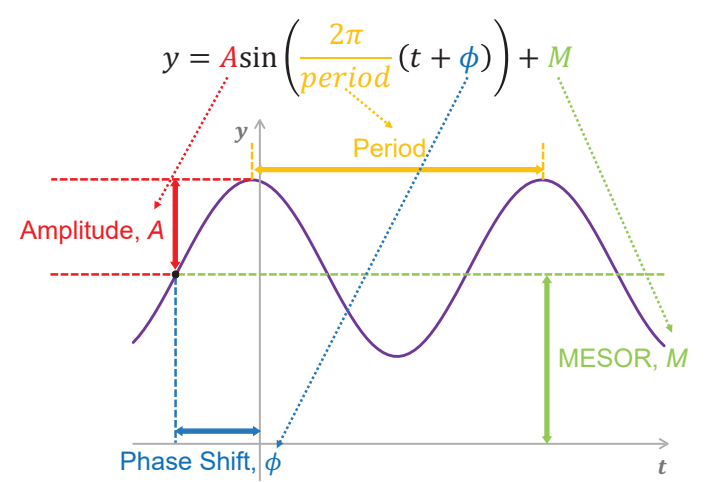

(b)

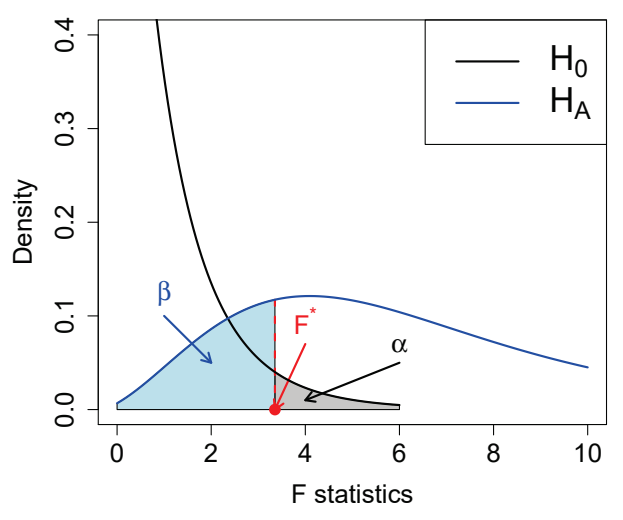

(c)

Figure 1. (a) Annual number of publications on PubMed that contain the keywords "circadian/clock" and one of the following omics type: "ChIPseq", "Metabolomics", "Methylation", "Proteomics", "Transcriptomics". (b) The sinusoidal wave curve underlying circadian rhythmicity power calculation framework. (c) The relationship between power and type I control in detecting circadian rhythmicity. The black curve represents the density function of the F statistics under the null distribution (no circadian pattern); the blue curve represents the density function of the $\mathrm{F}$ statistics under the alternative distribution. The red dashed line represents the decision boundary (i.e., $F^{*}$ ) such that the type I error rate is controlled at $\alpha$ (shaded gray). The corresponding type II error $\beta$ is the area with lightblue color and the detection power is $1-\beta$. 
collecting, calculating, and summarizing the intrinsic effect sizes of existing human and animal studies, which serves as a useful reference resource when no pilot data is available; and (vi) providing an open-source $\mathrm{R}$ package.

The superior performance of our method is demonstrated in comprehensive simulation studies, as well as multiple transcriptomic applications in human and mouse. We demonstrate the performance of CircaPower using gene expression data throughout this manuscript, but our method is also applicable in other types of omics data, including but not restricted to ChIP-Seq, DNA methylation, proteomics, metabolomics, and even non-omics data.

\section{MATERIALS AND METHODS}

The CircaPower framework assumes the relationship of the expression level of a gene and the Zeitgeber time (ZT) fits a sinusoidal wave curve, and is based on the $\mathrm{F}$ statistics of a cosinor model (7). Below we introduce the model notations, the construction of the F statistics, the null and alternative distribution of the F statistics, the closed-form formula for circadian power calculation, and factors affecting the power calculation of circadian rhythmicity detection.

\section{Notations and basic model}

As illustrated in Figure 1b, denote $y$ as the expression value for a gene; $t$ as the ZT; $M$ as the MESOR (Midline Estimating Statistic Of Rhythm, a rhythm-adjusted mean); $A$ as the amplitude. $\omega$ is the frequency of the sinusoidal wave, where $\omega=\frac{2 \pi}{\text { Period }}$. Without loss of generality, we set period $=24$ hours to mimic the diurnal period. $\phi$ is the phase shift of the sinusoidal wave curve. Whenever there is no ambiguity, we will omit the unit "hours" in period, phase, and other related quantities. Due to the periodicity of a sinusoidal wave, $\left(\phi_{1}, \phi_{2}\right)$ are not identifiable when $\phi_{1}=\phi_{2}+24$. Therefore, we will restrict $\phi \in[-6,18) . \phi$ is not intuitive to read from a sinusoidal wave (See Figure 1b), and a closely related quantity is the peak time $t_{P}$. The connection between $\phi$ and $t_{P}$ is that $\phi+t_{P}=6 \pm 24 N$, where $N$ is an arbitrary natural number.

For a given sample $i(1 \leq i \leq n, n$ is the total number of samples), denote by $y_{i}$ the expression value of a gene and $t_{i}$ the observed ZT. We assume the following sinusoidal wave function:

$y_{i}=A \sin \left(\omega\left(t_{i}+\phi\right)\right)+M+\varepsilon_{i}$,

where $\varepsilon_{i}$ is the error term for sample $i$; we assume $\varepsilon_{i}$ 's are identically and independently distributed (i.i.d.) from $\varepsilon_{i} \sim N\left(0, \sigma^{2}\right)$, where $\sigma$ is the noise level. To benchmark the goodness of sinusoidal wave fitting, we define the coefficient of determination $R^{2}=1-\frac{R S S}{T S S}$, where $R S S=\sum_{i=1}^{n}\left(y_{i}-\right.$ $\left.\hat{y}_{i}\right)^{2}, T S S=\sum_{i=1}^{n}\left(y_{i}-\bar{y}\right)^{2}, \hat{y}_{i}=\hat{A} \sin \left(\omega\left(t_{i}+\hat{\phi}\right)\right)+\hat{M}, \bar{y}=$ $\sum_{i} y_{i} / n$, with $\hat{A}, \hat{\phi}$, and $\hat{M}$ being the fitted value for $A, \phi$, and $M$ in Equation 1 under least squared loss. $R^{2}$ ranges from 0 to 1 , with 1 indicating perfect sinusoidal wave fitting, and 0 indicating no fitting at all. Equivalently, we could re-write Equation 1 as

$y_{i}=E \sin \left(\omega t_{i}\right)+H \cos \left(\omega t_{i}\right)+M+\varepsilon_{i}$, where $E=A \cos (\omega \phi)$, and $H=A \sin (\omega \phi)$, which turns into a linear regression problem.

\section{Analytical power calculation}

According to linear model theories, the F statistics for the circadian model in Equation 1 can be derived as:

$$
F^{s t a t}=\frac{\frac{T S S-R S S}{r-1}}{\frac{R S S}{n-r}}
$$

where $n$ is number of independent samples, $r=3$ is number of parameters (i.e., $A, \phi$, and $M$ in Equation 1).

The null hypothesis is that there is no circadian rhythmicity. In other words, $A=0$ in Equation 1 (equivalently, $E=H=0$ in Equation 2). Under the null hypothesis,

$$
F^{s t a t} \sim f_{0}(\cdot \mid 2, n-3)
$$

where 2 and $n-3$ are the degrees of freedom of the $F$ distribution, 0 in $f_{0}$ indicates this is a regular $F$ distribution with non-centrality parameter 0 (32).

The alternative hypothesis is that there exists a circadian rhythmicity pattern. In other words, $A \neq 0$ in Equation 1 (equivalently, $E \neq 0$ or $H \neq 0$ in Equation 2). Under the alternative hypothesis, the $\mathrm{F}$ statistics follows a non-central $\mathrm{F}$ distribution, with non-centrality parameter $\lambda=\frac{A^{2}}{\sigma^{2}} \sum_{i} \sin ^{2}\left(w\left(t_{i}+\phi\right)\right)$. The non-centrality parameter $\lambda$ controls the location parameter of the non-central $F$ distribution. A larger $\lambda$ will lead to a larger expected value (i.e., mean value) of the non-central $F$ distribution.

$$
F^{s t a t} \sim f_{\lambda}(\cdot \mid 2, n-3),
$$

where 2 and $n-3$ are the degrees of freedom of the $F$ distribution, $\lambda$ in $f_{\lambda}$ indicates this is a non-central $F$ distribution with non-centrality parameter $\lambda$. The proof is given in Supplementary Section 1.

Figure 1c shows the relationship between the null and alternative distributions. We further denote $F^{*}$ as the decision boundary, such that we declare a gene to be rhythmic if $F^{\text {stat }} \geq F^{*}$. By assuming the type I error rate corresponding to the decision boundary $F^{*}$ is $\alpha$, we have $\alpha=1-$ $F_{0}\left(F^{*} \mid 2, n-3\right)$, where $F_{0}\left(x \mid d f_{1}, d f_{2}\right)$ represents cumulative density function of $f_{0}\left(\cdot \mid d f_{1}, d f_{2}\right)$ evaluated at $x$. The power at the decision boundary $F^{*}$ is $1-\beta=1-F_{\lambda}\left(F^{*} \mid 2, n-3\right)$, where $F_{\lambda}\left(x \mid d f_{1}, d f_{2}\right)$ represents cumulative density function of $f_{\lambda}\left(\cdot \mid d f_{1}, d f_{2}\right)$ evaluated at $x$.

As shown in Figure 1c, the non-centrality parameter $\lambda$ controls the degree of separation of the null distribution $f_{0}$ and the alternative distribution $f_{\lambda}$. The larger the $\lambda$ is, the more likely the alternative distribution will be away from the null distribution, and the higher power a gene will achieve. We thus define $\lambda$ as the total effect size for the circadian power calculation. By inspecting the total effect size $\lambda=\frac{A^{2}}{\sigma^{2}} n \frac{1}{n} \sum_{i=1}^{n} \sin ^{2}\left(w\left(t_{i}+\phi\right)\right)$, this noncentrality parameter can be decomposed into three parts: (i) sample size $n$, (ii) intrinsic effect size $r=A / \sigma$ (closely relate to the goodness of fit statistics $R^{2}$ ), and (iii) sampling design 
effect $d=\frac{1}{n} \sum_{i=1}^{n} \sin ^{2}\left(w\left(t_{i}+\phi\right)\right)$. Therefore, $\lambda=n d r^{2}$. We then discuss the impact of each of these components on the circadian rhythmicity power calculation and show their impact by simulation in the Results section.

Sample size Fixing $d$ and $r$, a larger sample size $n$ will result in a larger total effect size $\lambda$, and achieve a higher statistical power. This is not a surprising conclusion since the sample size is an important factor in all power calculation procedures.

Intrinsic effect size Intuitively, a larger circadian amplitude $A$ with smaller residual variability $\sigma$ will lead to a better sinusoidal curve fitting (i.e. larger $R^{2}$ ). Our formula suggests that circadian fitting parameters $A$ and $\sigma$ work together as an intrinsic effect size $r(r=A / \sigma)$ and has a quadratic effect on the total effect size $\lambda$. This implies the two curve fitting parameters that need to be specified in conventional Monte-Carlo based power calculation can be reduced to one parameter $r$ (shown in the Results section).

Sampling design effect The sampling design effect $d=$ $\frac{1}{n} \sum_{i=1}^{n} \sin ^{2}\left(w\left(t_{i}+\phi\right)\right)$ is more complicated, because it involves the collected ZT $t_{i}$ from each individual, and the unknown parameter phase shift $\phi$. In general, given an arbitrary circadian sampling design, we need to estimate the circadian phase shift $\phi$ before performing power calculation. Fortunately, the power calculation for the evenly-spaced sampling design is independent of the phase value (i.e., phaseinvariant). For example, (16) recommended a collection of 12 time points (every 2 hours) per cycle across 2 full cycles, which belongs to the evenly-spaced sampling design. Such active design is commonly seen in animal studies or human blood studies, where researchers can control the exact time to sacrifice the animal or to collect blood. The following theorem (phase-invariant property) shows that the sampling design effect $d$ is a constant under the one-period one-sample evenly-spaced design. In other words, the ZT points are spread within one period, and there is only one sample per time point.

THEOREM 1 (Phase-invariant property - one-period one-sample). Assuming there is a total of $n$ ZT points $t_{i}(1 \leq$ $i \leq n)$ within a circadian period $2 \pi / \omega$, which are ordered such that $t_{i}<t_{i+1}$ for all $1 \leq i \leq n-1$. If $n \geq 3$, and $t_{i}$ is evenlyspaced over the period (i.e., $t_{i+1}-t_{i}=\bar{C}$ for all $1 \leq i \leq n-1$, $C>0$ is a fixed time interval, $\left.\left(t_{1}+2 \pi / \omega\right)-t_{n}=C\right)$, then regardless of the value for $\phi$, we have

$$
\frac{1}{n} \sum_{i=1}^{n} \sin ^{2}\left(w\left(t_{i}+\phi\right)\right)=\frac{1}{2}
$$

The above theory shows sampling design effect is phaseinvariant for the one-period one-sample evenly-spaced design. The proof is given in the Supplementary Section 2. It can immediately be extended to the following corollary for multiperiod (two or more cycles), multi-sample, evenly-spaced design.

COROLLARY 1 (Phase-invariant property - multi-period multi-sample). For multi-period multi-sample evenly-spaced design, the sampling design effect is phase-invariant.

$$
\frac{1}{n} \sum_{i=1}^{n} \sin ^{2}\left(w\left(t_{i}+\phi\right)\right)=\frac{1}{2}
$$

This is because the multi-period multi-sample evenlyspaced design just replicates the one-period one-sample evenly-spaced design, therefore the average of them remains to be $1 / 2$.

\section{Assumptions underlying the circadian modeling framework}

The proposed circadian modeling framework has two underlying assumptions: (i) the relationship between the expression level of a gene and the ZT forms a sinusoidal wave curve; (ii) the error terms of each sample on top of the sinusoidal wave curve follows independent and identical Gaussian distribution. In the Result section, we not only demonstrate that the $\mathrm{F}$ statistics is robust against various types of violation of model assumptions including (i) heavy tail error distributions, (ii) existence of outliers, and (iii) non-independent error distributions, but also discuss the implication of sinusoidal assumption on sampling design.

\section{Alternative power calculation method by Monte-Carlo simulation}

Without the proposed analytical method CircaPower, a conventional method for circadian detection power calculation is by Monte-Carlo simulation (MC), which assumes known $A$, $\phi, M, \sigma$ and $t_{i}, 1 \leq i \leq n$ in Equation 1. Detailed algorithm for $\mathrm{MC}$ is described as following:

1. Given the ZT $t_{i}^{\prime} s$ for $n$ samples $(1 \leq i \leq n)$ and key parameters $(A, \phi, M$, and $\sigma$ ), we simulate gene expression $y_{g i}$ based on Equation 1, where $1 \leq g \leq G$ is the gene index and $G$ is the total number of genes.

2. We apply the cosinor method (7) to derive the rhythmic p-value $p_{g}$ for each gene $g(1 \leq g \leq G)$. Given a prespecified alpha level $\alpha$, the MC power is calculated as $\frac{\sum_{g} I\left(p_{g} \leq \alpha\right)}{G}$. We set the alpha level $\alpha=0.001$ and simulate $G=10,000$ genes throughout the simulation studies in this manuscript, unless otherwise specified.

Both the CircaPower and the MC rely on the F statistics for rhythmicity detection, which is generally robust against violation of Gaussian assumptions (shown in the Result section). However, the CircaPower has several obvious advantages over the MC. First of all, the explicit representation of total effect size in CircaPower provides insights on the three determining factors $(n, r, d)$ in circadian detection power calculation while it is hard for MC simulation to determine selections and trends on the many parameters $(A, \phi, M, \sigma$, and $\left.t_{i}, 1 \leq i \leq n\right)$. In addition, our simulation in the Result section shows the closed-form solution by CircaPower is at least 10,000 folds faster than the MC approach. More importantly, even though both approaches can calculate power given sample size, only CircaPower can directly solve the inverse problem of deriving the smallest sample size 


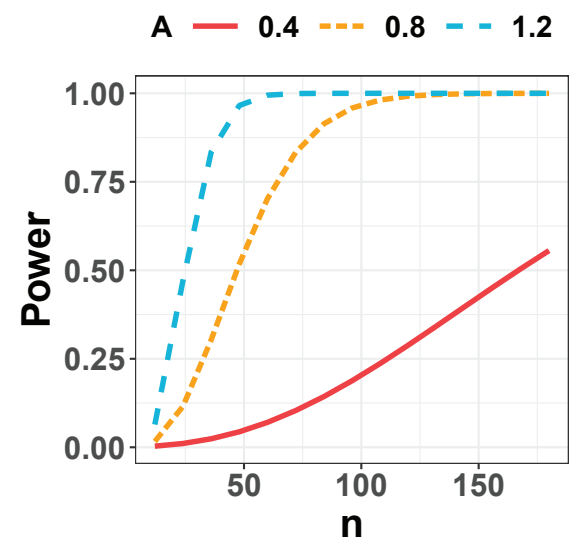

(a)

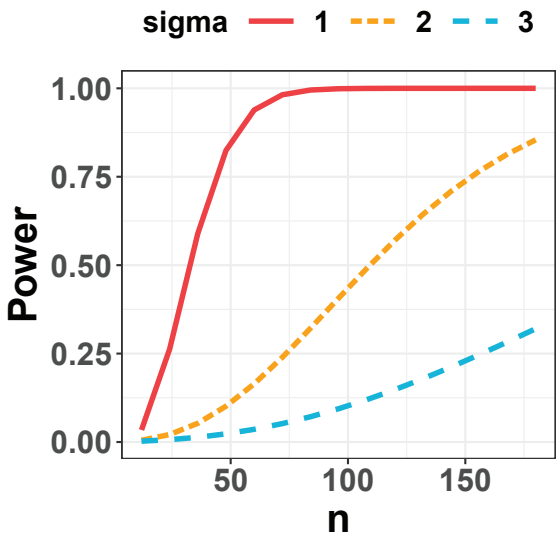

(b)

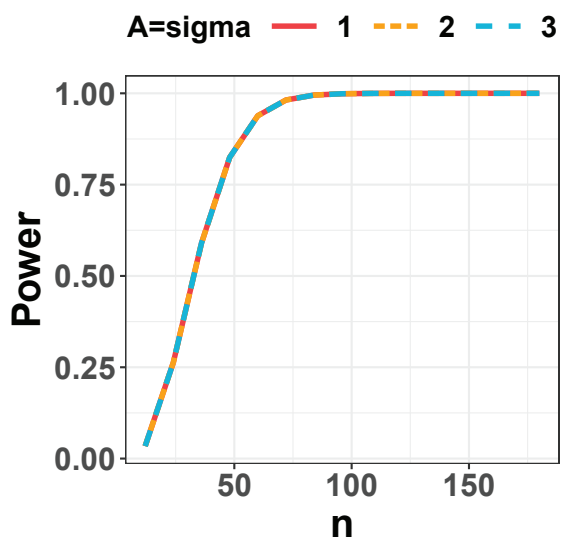

(c)

Figure 2. Impact of the intrinsic effect size $r=A / \sigma$ on circadian power calculation. (a) shows the impact of amplitude (i.e., $A=0.4,0.8,1.2$ ) when the noise level is fixed at $\sigma=1$; (b) shows the impact of noise level (i.e., $\sigma=1,2,3$ ) when the amplitude is fixed at $A=1$. (c) shows the impact of intrinsic effect size by co-varying $A$ and $\sigma$ simultaneously (i.e., $A=1,2,3, \sigma=1,2,3)$ while keeping their ratio as a constant (i.e., $r=A / \sigma=1$ ).

meeting the desired detection power, while MC needs repeated interpolation to obtain an answer.

\section{RESULTS}

Throughout simulations and real applications, we control type I error $\alpha=0.001$ for circadian power calculations to account for potential multiple comparisons.

\section{Impact of sample size and intrinsic effect size in simulation}

To evaluate the impact of sample size $n$ and intrinsic effect size $r=A / \sigma$, we assume the $\mathrm{ZT}$ is from the oneperiod one-sample evenly-spaced design, which enjoys the phase-invariant property $(d=1 / 2$, Theory 1$)$. The impact of sampling designs will be discussed in next section. Since phase shift $\phi$ has no impact on power calculation under the phase-invariant property, we fix $\phi=0$ unless otherwise specified. We vary $n=12,24, \ldots, 180$ to show the sample size effect (Figure 2). As expected, a larger $n$ will lead to larger statistical power regardless of the choice of the intrinsic effect size $r$.

To examine the impact of intrinsic effect size $r=A / \sigma$. We first vary $A=0.4,0.8,1.2$ while fixing $\sigma=1$, which is also equivalent to varying $r=0.4,0.8,1.2$. As shown in Figure 2a, we observe a larger $A$ (or larger $r$ ) will result in larger statistical power. We then vary $\sigma=1,2,3$ while fixing $A=$ 1 , which is also equivalent to varying $r=1,0.67,0.33$. As shown in Figure 2b, we observe a larger $\sigma$ (or smaller $r$ ) will result in smaller statistical power. We finally co-vary $A$ and $\sigma$ simultaneously (i.e., $A=1,2,3$ and $\sigma=1,2,3$ ) while fixing their ratio as a constant (i.e., $r=A / \sigma=1$ ). As shown in Figure 2c, we observe the power trajectories remain identical as long as the $r$ is fixed as a constant (i.e., $r=1$ ). These results demonstrate that the proposed $r=A / \sigma$ is sufficient to capture the effect size information in relation to the goodness-of-fit of the cosinor model.

\section{Impact of sampling design in simulation}

In this section, we explore the impact of different sampling designs on circadian power calculation using CircaPower. Since the sample collection scheme for active design and passive design are quite different, we will discuss them separately. For all these different sampling design schemes, we vary the intrinsic effect size $r=0.4,0.8,1,1.2$. Due to the fact that not all designs have the phase-invariant property, we also vary the phase shift $\phi=0,3,6$.

For a typical active design, researchers usually need to control (i) the number of ZT points per cycle, (ii) the number of replicated samples at each time point within a cycle, and (iii) the number of cycles. Because of the periodicity property of the sinusoidal curve in the cosinor model, the number of replicated samples at each time point within a cycle and the number of cycles are equivalent in the sense that 2 replicated samples at each time point across 1 cycle will result in the same statistical estimation with only one sample at each time point across 2 cycles. Therefore, for the ease of discussion, we summarize the following two key parameters for an active design: (i) number of ZT points $N_{T}$ per cycle; (ii) total number of samples $n$. The number of replicated samples (at the same ZT across all cycles) could be calculated as $n / N_{T}$.

We denote the active design scheme with $N_{T}$ ZT points per cycle as FixTime $N_{T}$. To investigate the impact of $N_{T}$ on the circadian power calculation, we perform simulations by varying $N_{T}=3,4,6, n . N_{T} \geq 3$ is necessary for the phaseinvariant property (Theory 1 and Corollary 1 ). When $N_{T}=$ $n$, the FixTime- $n$ design is the same as the one-period one-sample evenly-spaced design (denoted as the EvenSpace design). Figure 3 a shows the power trajectories of these evenly-spaced sampling designs. We observe that (i) the power curves are the same regardless of the phase shift value, confirming the phase-invariant property of the evenly-spaced sampling design; (ii) the power trajectories for different $N_{T}^{\prime} s$ are also identical, which implies that under evenly-spaced sampling design, the detection power only depends on the total number of samples $n$ but not the $N_{T}$ as long as it is greater or equal to 3 . Note that these arguments on the number of 


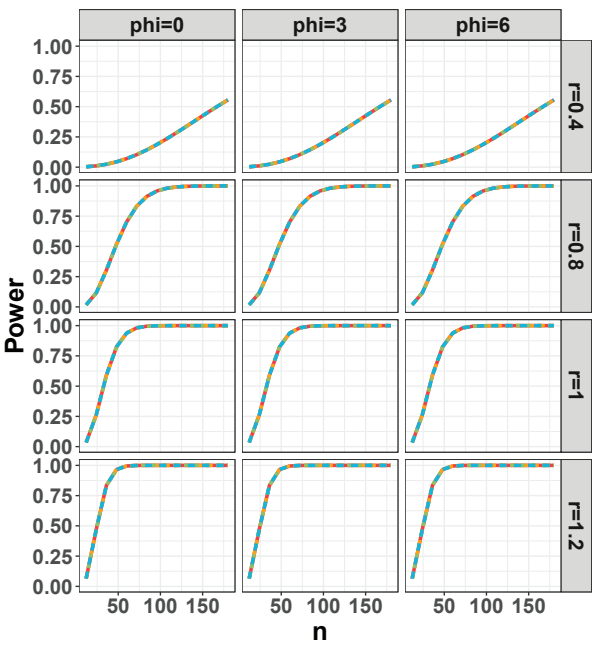

(a)

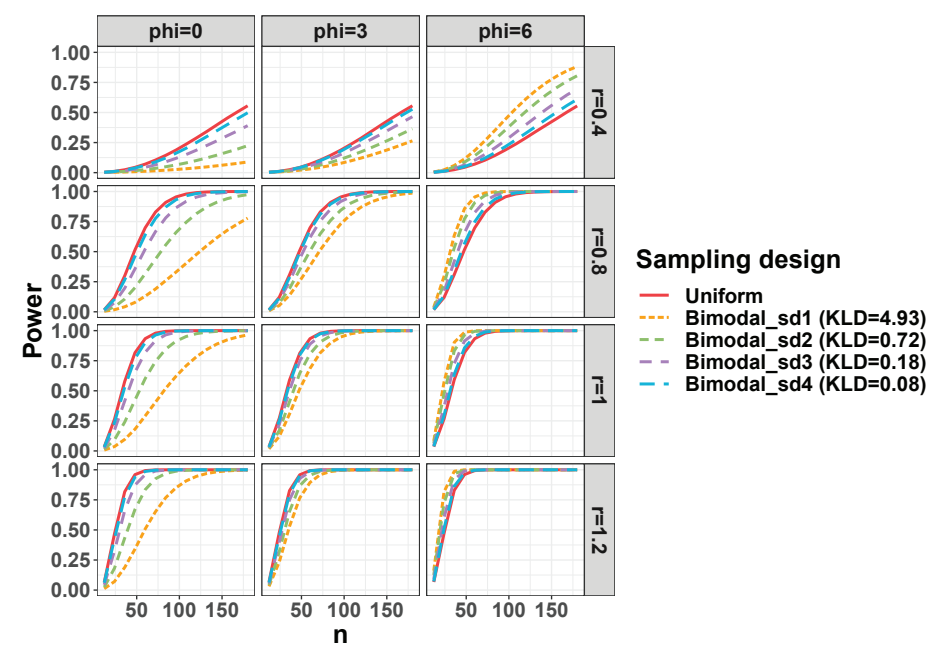

(b)

Figure 3. Sampling design effect on circadian power calculation. (a) shows the sampling design effect for active design; (b) shows the sampling design effect for passive design.

ZT points $N_{T}$ are purely based on the statistical power given sinusoidal wave assumption. In reality, less number of time points may result in unstable circadian curve fitting (See next section for details).

For passive designs, the collection of the ZT cannot be controlled. We therefore simulate $t_{i}^{\prime} s$ from (i) uniform distribution (uniform design): $\quad t_{i} \stackrel{i i d}{\sim} \operatorname{UNIF}(0,24)$; $\quad$ and (ii) bimodal Gaussian distributions (bimodal designs): $t_{i} \sim p_{i} N(7, s d)+\left(1-p_{i}\right) N(17, s d) ; \quad p_{i} \sim \operatorname{Bernulli}(0.5)$. We vary the standard deviation of bimodal components to be $s d=1,2,3,4$ whose Kullback-Leibler divergence (KLD) against the uniform distribution are 4.93,0.72,0.18,0.08 respectively, suggesting smaller deviation from uniform as $s d$ increases. KLD is used as a relative measurement to benchmark the divergence between the uniform and the bimodal distributions with various spread. Figure $3 b$ shows that for the uniform design, the power trajectory is almost phase-invariant. This is expected since the uniform distribution is a random realization of the evenly-spaced sampling design, and the impact of phase on the individual $t_{i}$ will average out. The bimodal designs show phase-dependent circadian power trajectories and the level of phase influence increases as the distribution deviates more from uniform (i.e., larger KLD). Specifically, Figure $3 b$ shows that the power loss of bimodal designs when $\phi=0,3$ is significant when KLD is 0.72 or greater (green and yellow curve) while negligible when KLD is only 0.18 or smaller (purple and blue curve). In fact, since the phase shift impacts the sampling design through $d=\frac{1}{n} \sum_{i=1}^{n} \sin ^{2}\left(w\left(t_{i}+\phi\right)\right)$; it achieves higher power if the mode of the ZT distribution occurs at the underlying peak/trough time. In real omics applications, it is expected that circadian genes have different phase shift values over the day. If the collected ZT distribution is far away from the uniform distribution, the detection power of each circadian gene would be affected by its phase shift differently across the genome.

\section{Larger number of time points per cycle to ensure sinusoidal curve fitting}

From the perspective of circadian power calculation, Figure $3 \mathrm{a}$ implies that the evenly-spaced sampling design is phaseinvariant as long as the number of time points per cycle $N_{T}$ is greater or equal to 3 . This is further corroborated by Corollary 1 , in which the sampling design effect $d=$ $1 / 2$ for any evenly-spaced sampling design. However, in the perspective of curve fitting, smaller number of time points may not necessarily guarantee the goodness-of-fit for a sinusoidal curve, resulting in potentially false positive findings.

To demonstrate this, we simulate expression data from the sinusoidal model and perform non-parametric curve fitting to explore the impact of number of $N_{T}$ on the goodness-of-fit for a sinusoidal wave. To be specific, we first choose the ZT points within a cycle to be $N_{T}=2,3,4,6,8,12$. Then for each $N_{T}$, we simulate $n=48$ samples, and evenly allocated them at $2 N_{T}$ time points across 2 full cycles (every $24 / N_{T}$ hours, from $-12 \mathrm{~h}$ to $36 \mathrm{~h}$ ), resulting in $24 / N_{T}$ samples at each time point. The expression values of samples at each time point $t_{j}$ are simulated independently from Equation 1, where we set $A=1, M=0$, and $\sigma=1$. The LOESS regression is then used to fit a smooth curve through the data points. The rationale for 2 full cycles is to improve the boundary behavior of the curve fitting within one cycle. Such LOESS regression represents the smooth curve fitting without the sinusoidal assumption, which could reflect the minimum of $N_{T}$ that is necessary to capture the sinusoidal wave curve. In addition, to evaluate the effect of the phase shift, we set $\phi=0,1, \ldots, \min \left(24 / N_{T}-1,6\right)$.

The data points and fitted smooth curves in one circadian cycle $[0,24]$ are shown in Figure S1. When the number of ZT points per cycle $N_{T}$ is 2 or 3 , it is uncertain whether the underlying curve fitting is a sinusoidal wave. When $N_{T}=4$, the curve shape is roughly sinusoidal although not smooth. When $N_{T}$ increases to 6 or more, the curve fitting is stable and almost identical to the underlying sinusoidal wave. Therefore, considering both circadian power calculation and smooth 
curve fitting, our results suggest $N_{T}=4$ to be the minimum number of ZT points per cycle to estimate the circadian curve shape. But in general, our results imply at least $6 \mathrm{ZT}$ points per cycle to fully capture the circadian rhythmicity pattern, which is commonly adopted in the literature.

\section{CircaPower achieves same power value at a much faster speed compared with the MC approach}

We compare CircaPower with the MC algorithm described in Method section. Specifically, we simulate data for MC following Equation 1 in the cosinor model.

$$
y_{i}=A \sin \left(\omega\left(t_{i}+\phi\right)\right)+M+\varepsilon_{i},
$$

where the error term $\varepsilon_{i}$ 's are independently identically distributed (iid), i.e., $\varepsilon_{i} \sim \mathrm{N}\left(0, \sigma^{2}\right)$. For both methods, the ZT points are simulated from one-period one-sample evenlyspaced design for the ease of discussion, which enjoys the phase-invariant property $(d=1 / 2$, Theory 1$)$. Since phase shift $\phi$ and MESOR $M$ has no impact on circadian detection power calculation in this case, we fix $\phi=0$ and $M=10$. We evaluate their power derived at a grid of $A=(0.4,0.8,1,1.2)$ and $\sigma=(1,2,3,4)$. Note that for CircaPower, we only need the underlying parameters $A, \sigma, t_{i}$, and $\phi$ to perform power calculation, which does not rely on the simulated dataset. We simulate the data for the purpose of evaluating the MC algorithm.

Figure S2 shows the power calculation results for both CircaPower and the MC. We observe that the power calculated from CircaPower is almost identical to the MC, corroborating the correctness of the closed-form solution in CircaPower.

In terms of computing time, to generate all the results in Figure S2, it takes 1.84 seconds for the CircaPower using 1 CPU thread on a regular PC (8th Gen Intel Core i5-8250U Quad-Core processor, $1.60 \mathrm{GHz}$ ), while it requires 8 hours for the MC using the same computing resource. With parallel computing, the computing time reduces to 0.13 seconds for CircaPower using 40 CPU threads on a Linux server (Intel Xeon Gold 6130, 2.10GHz), while it still needs 24 minutes for the MC.

\section{F statistics is robust against violations of the $i$ id Gaussian assumption}

To examine the robustness of our method when the iid Gaussian assumption is violated, we investigate the type I error control of $F$ statistics in the following scenarios: (i) heavy tail error distribution (i.e., student t distribution); (ii) existence of outliers; (iii) non-independent Gaussian errors. For all these simulations, $G=10,000$ noisy genes are simulated with error term $\varepsilon_{g i}$ 's specified above. By declaring circadian rhythmicity at $5 \%$ nominal $\alpha$ level, we will evaluate the actual type I error rate of the F test from the cosinor model. Since CircaPower is built on the F statistics for rhythmicity detection it will be benchmarked as robust if the actual type I error rate is close to the nominal $\alpha$ level. Since our goal is to evaluate the type I error rate control, which does not involve in any multiple testing issue, we directly use 5\% nominal $\alpha$ level.

Heavy tail error distribution Instead of sampling the error term $\varepsilon_{g i} \stackrel{i i d}{\sim} N\left(0, \sigma^{2}\right), 1 \leq g \leq G$ and $1 \leq i \leq n$, we sample $\varepsilon_{g i} \stackrel{i i d}{\sim} t(d f)$, where $t(d f)$ is the student $\mathrm{t}$ distribution with degree of freedom $d f$. In general, the smaller the $d f$ is, the heavier tail the error distribution is. When $d f=2$, the error distribution becomes the Cauchy distribution, and when $d f \rightarrow \infty$, the error distribution converges to standard Gaussian distribution (i.e., $N\left(0, \sigma^{2}\right)$ ). To evaluate the impact of heavy tail error distribution on CircaPower, we simulate a grid of $d f=(2.5,3,5, \infty)$. Figure S3a shows that when there is no or mild violation of the Gaussian assumption (i.e., $d f=\infty$ or $d f=5)$, the cosinor method achieves accurate type I error control (i.e., $5 \%$ ). When there is moderate to severe violation of the Gaussian assumption (i.e., $d f=3$ or $d f=2.5$ ), type I error rate is only slightly conservative (i.e., below the nominal $\alpha=0.05)$. Putting together, the type I error rate of the cosinor method can be correctly controlled against the heavy tail error distribution. And thus, the CircaPower is robust against heavy tail error distributions.

Existence of outliers To evaluate the impact of outliers on type I error control, we replace $q \%$ of the expression values with outliers, where $q=(5,10,20)$. To be specific, for a gene $g$, there is $q \%$ chance that the expression level is simulated from $y_{g i} \stackrel{i i d}{\sim} \operatorname{UNIF}(M-A, M+A)$; and $1-q \%$ chance that the expression level is simulated independently based on Equation 1 under $H_{0}: A=0$ (i.e., $y_{g i}=M+\varepsilon_{g i}$, $\left.\varepsilon_{g i} \stackrel{i i d}{\sim} N\left(0, \sigma^{2}\right)\right)$. Figure $\mathrm{S} 3 \mathrm{~b}$ shows that the cosinor method achieves accurate nominal type I error rate control (i.e., 5\%), showing robustness to outliers. And thus, the CircaPower is robust against the existence of outliers.

Correlated gene structure Instead of assuming all genes are independent, we simulate every 50 genes as a gene module with correlation coefficient $\rho$. The error term for each gene module $\boldsymbol{\varepsilon}_{50} \sim N\left(\mathbf{0}, \boldsymbol{\Sigma}_{50}\right)$ where $\boldsymbol{\Sigma}_{50}$ is a symmetric matrix with diagonal elements being $\sigma^{2}$ and off-diagonal elements being $\sigma^{2} \rho$. We simulate a grid of $\rho=(0,0.25,0.5,0.75)$. Figure S3c shows that when genes are correlated, the cosinor method maintains accurate nominal type I error rate control (i.e., 5\%), indicating that the CircaPower is robust against the dependency assumption.

\section{CircaPower for human studies with passive design}

We investigate the power trajectories of human studies using three human post-mortem brain transcriptomic studies (Chen (5), Seney (39) and Ketchesin (20)) with different time of death distribution. Detailed descriptions of each dataset can be referred in the original papers. Briefly, Chen (5) and Seney (39) performed gene expression circadian analysis using microarray $(n=147)$ and RNA-seq $(n=104)$ respectively using pre-frontal cortex tissues; and Ketchesin (20) performed RNA-seq gene expression circadian analysis with $n=59$ participants using dorsal and ventral striatum tissues.

Since investigators in these human post-mortem brain studies have no control of sample collection time (i.e., time of death) and can only accept passive sampling design, the detection power curves are not phase-invariant, which is unlike evenly-spaced active sampling design. For all three human studies, the tissue collection time is delayed with a post-mortem interval (PMI) after time of death so the recorded 
time is only a rough estimate. However, all these studies have reported successful circadian gene detection so we will ignore this factor and consider it as part of the model uncertainty $\sigma$. For all these three studies, we use their time of death to characterize the sampling design factor $d$.

To estimate the intrinsic effect sizes from the three brain studies, we apply the cosinor method (7) to identify genes with rhythmic patterns and obtain estimates for their amplitude $\hat{A}$ and noise level $\hat{\sigma}$. We estimate the intrinsic effect sizes $\hat{r}=\hat{A} / \hat{\sigma}$ using the 7 core circadian genes, or the top 100 significant rhythmic genes (ranked by $\mathrm{p}$-values from the cosinor method). The 7 core circadian genes include Arntl, Dbp, Nrld1, Nrld2, Perl, Per2, and Per3, which showed persistent circadian pattern across 12 mouse tissues (49). The Homo sapiens section of Table 1 shows the estimated intrinsic effect sizes for: (i) median $r$ of the 7 core circadian genes; (ii) minimum $r$ of the 100 most significant circadian genes. The estimated intrinsic effect sizes for these three human studies range between 0.44 and 1.06 .

To demonstrate the power trajectories in real data, we vary intrinsic effect sizes $r=0.4,0.6,0.8,1$, which roughly cover the estimated range of the intrinsic effect sizes in the postmortem brain studies. The ZT points are sampled 1000 times from the kernel density estimated from the observed timeof-death distributions in these three studies and a uniform distribution (See top panels of Figure 4a). Since the sampling design factor $d$ is not a constant in passive design and the power calculation is not phase-invariant, we vary phase shift $\phi=0,1,2,3, \ldots, 12$ and use a confidence band to represent the range of power achieved across phase shifts (See bottom panels of Figure 4a). For each scenario (i.e., fixed $r, n$ and $\phi$ ), the mean power among the 1000 times repetitions is reported.

As expected, larger sample size $n$ and larger intrinsic effect size $r$ lead to a larger circadian power and the power trajectory is almost phase-invariant for the uniform sampling design with band width close to 0 , but not for the passive sampling designs from the three human studies. As discussed, the power will depend on the relationship between the mode of the ZT distribution and the underlying peak/trough time. To further demonstrate the impact of phase on the nonuniform distribution for ZT, we fix $n=120, r=0.6$, while varying $\phi=0,1,2,3, \ldots, 12$. As shown in Figure 4b), the power trajectories fluctuates across different $\phi^{\prime} s$ when samples are draw from non-uniform distributions in the three postmortem studies, while the trajectory stays almost the same for uniformly distributed ZT. However, since the KLDs of the kernel densities estimated from the Chen, Seney and Ketchesin are relatively low (i.e., $0.12,0.17,0.14$ ) in the context of bimodal designs, the variation of power as a result of phase shift is small, with $4.8 \%, 3.5 \%$, and $8.3 \%$ maximum drop, respectively.

\section{CircaPower for animal studies with with active sampling design}

We next examine the power trajectories of actively designed mouse studies using 14 mouse gene expression circadian data $(1,3,6,11,14,17,28,29,30,33,34,36,41,49)$ from 20 types of tissues, including adipose, adrenal gland, aorta, atrium, brainstem, brown fat, cerebellum, cerebral cortex, colon, epidermal, fibroblast, heart, hypothalamus, kidney, liver, lung, muscle, satellite, ventricle and white fat. Sample sizes of each study tissue are shown in Table 1. To estimate the intrinsic effect sizes of these tissues, we similarly apply the cosinor method (7) to identify genes with rhythmic patterns and obtained estimates for their amplitude $\hat{A}$ and noise level $\hat{\sigma}$. The estimated intrinsic effect sizes for the median $r$ of the 7 core circadian genes and the minimum $r$ of the top 100 significant circadian genes are shown in the Mus musculus section of Table 1, ranging from 0.96 to 6.33 , a much larger magnitude than previous human studies. This is reasonable since human studies are usually more heterogeneous in terms of genetics and environmental background. We thus fix the intrinsic effect sizes to be $r=1,2,3,4$ in our subsequent power calculation. Since these experiments employ an evenlyspaced active sampling design, the sampling design factor is a constant (i.e., $d=1 / 2$, Corollary 1 ) regardless of the phase value.. As a result, we employ the one-sample one-period evenly-spaced design (See left panel of Figure 5) for the purpose of power calculation. By further assuming the alpha levels to be $\alpha=0.05,0.01,0.001$, the power trajectories with respect to sample size $n$ is shown in Figure 5 (right panel).

\section{Case study: circadian power calculation using mouse pilot dataset}

To demonstrate how to perform circadian power calculation using pilot dataset from scratch, we utilize a circadian gene expression data in mouse with skeletal muscle, which is part of the mouse pan-tissue gene expression circadian microarray data (49). Detailed description of this dataset has been described in previous literature (13). Briefly, 24 mouse muscle samples were collected (every 2 hours) across 2 full cycles. With this pilot data, we perform genome-wide circadian rhythmicity detection using the cosinor method (7). Under $p<0.001$, we identify 716 significant genes showing circadian pattern. We similarly estimate the intrinsic effect sizes for: (i) median $r$ of the 7 core circadian genes; (ii) minimum $r$ of the top 100 significant circadian genes. The resulting intrinsic effect sizes are 3.58 and 2.23 respectively. By assuming different $\alpha$ to be $0.05,0.01,0.001$, the power curves with respect to sample size are shown in Figure S4. We observe that $n=12$ can achieve $97.1 \%$ and $50.5 \%$ detection power for the two intrinsic effect sizes at $\alpha=0.001$.

\section{DISCUSSION}

In this paper, we propose an analytical framework, CircaPower, to calculate the statistical power for circadian gene detection analysis. To the best of our knowledge, this is the first analytical method to perform circadian power analysis. In simulations, we not only demonstrate the CircaPower is fast and accurate, but also show that CircaPower is robust against violations of model assumptions. In real applications, we obtain the estimated intrinsic effect sizes from publicly available human and mouse transcriptomic circadian data and show the performance of CircaPower. When a user needs to perform power calculation without pilot data, information from these public data can be used as a reference resource to facilitate circadian power calculation. In case study, we also demonstrate circadian power calculation step-by-step given a pilot dataset. 
Table 1. Intrinsic effect sizes for public available transcriptomic circadian data, including 3 passively designed human postmortem brain studies and 14 actively designed mouse studies from 20 types of tissues. These data are processed using the cosinor method (7). Two types intrinsic effect sizes are used: (i) median $r$ of the 7 core circadian genes; (ii) minimum $r$ of the top 100 significant circadian genes. These intrinsic effect sizes can be used as a reference resource when investigators need to perform power calculation without any pilot data.

\begin{tabular}{|c|c|c|c|c|c|c|}
\hline Organism & Study & $\begin{array}{c}\text { Data } \\
\text { Availability }\end{array}$ & Tissue & $\begin{array}{l}\text { Sample } \\
\text { Size }\end{array}$ & $\begin{array}{c}\text { Median } r \\
\text { of the } 7 \text { core } \\
\text { circadian genes }\end{array}$ & $\begin{array}{c}\text { Minimum } r \\
\text { of the top } 100 \\
\text { circadian genes }\end{array}$ \\
\hline \multirow{6}{*}{$\begin{array}{l}\text { Homo } \\
\text { sapiens }\end{array}$} & \multirow{2}{*}{ Chen (5) } & \multirow{2}{*}{ GSE71620 } & Pre-frontal cortex (BA11) & 147 & 0.91 & 0.46 \\
\hline & & & Pre-frontal cortex (BA47) & 147 & 0.77 & 0.44 \\
\hline & \multirow{3}{*}{ Ketchesin (20) } & \multirow{3}{*}{ GSE160521* } & Striatum (NAc) & 59 & 0.71 & 1.06 \\
\hline & & & Striatum (caudate) & 59 & 1.04 & 0.82 \\
\hline & & & Striatum (putamen) & 59 & 0.83 & 1.02 \\
\hline & Seney (39) & $\begin{array}{l}\text { Common Mind } \\
\text { Consortium* }\end{array}$ & Pre-frontal cortex & 104 & 0.79 & 0.55 \\
\hline \multirow{40}{*}{$\begin{array}{c}\text { Mus } \\
\text { musculus }\end{array}$} & Aguilar-Arnal(1) & GSE49638 & Fibroblast & 18 & 2.38 & 2.94 \\
\hline & \multirow{2}{*}{ Bray(3) } & \multirow{2}{*}{ GSE10045 } & Atrium & 32 & 3.43 & 1.46 \\
\hline & & & Ventricle & 32 & 0.96 & 1.09 \\
\hline & Cho(6) & GSE34018 & Liver & 12 & 2.84 & 4.11 \\
\hline & Gerstner(11) & GSE78215 & Cerebral cortex & 34 & 2.46 & 2.18 \\
\hline & Hoogerwerf(14) & GSE10644 & Colon & 18 & 1.86 & 1.56 \\
\hline & \multirow{2}{*}{ Hughes (17) } & GSE11922 & Fibroblast & 48 & 1.27 & 1.02 \\
\hline & & GSE11923 & Liver & 48 & 2.00 & 2.77 \\
\hline & Mari(28) & GSE52333 & Liver & 18 & 4.11 & 3.39 \\
\hline & Masri (29) & GSE73222 & Liver & 18 & 3.83 & 2.47 \\
\hline & Masri (30) & GSE57830 & Liver & 36 & 2.69 & 2.20 \\
\hline & $\mathrm{Na}(33)$ & GSE11516 & Liver & 36 & 3.65 & 3.69 \\
\hline & Nikolaeva(34) & GSE27366 & Kidney & 12 & 2.39 & 2.61 \\
\hline & Paschos(36) & GSE35026 & Adipose & 12 & 2.48 & 2.58 \\
\hline & \multirow{2}{*}{ Solanas(41) } & \multirow{2}{*}{ GSE84580 } & Satellite & 24 & 3.64 & 2.30 \\
\hline & & & Epidermal & 20 & 5.03 & 2.62 \\
\hline & \multirow{24}{*}{ Zhang(49) } & \multirow{12}{*}{ GSE54650 } & Adrenal gland & 24 & 5.17 & 2.27 \\
\hline & & & Aorta & 24 & 5.55 & 2.29 \\
\hline & & & Brainstem & 24 & 3.90 & 2.07 \\
\hline & & & Brown fat & 24 & 5.05 & 2.72 \\
\hline & & & Cerebellum & 24 & 3.52 & 2.01 \\
\hline & & & Heart & 24 & 4.47 & 2.82 \\
\hline & & & Hypothalamus & 24 & 2.67 & 1.74 \\
\hline & & & Kidney & 24 & 6.33 & 3.65 \\
\hline & & & Liver & 24 & 3.51 & 3.67 \\
\hline & & & Lung & 24 & 5.78 & 3.47 \\
\hline & & & Muscle & 24 & 3.58 & 2.23 \\
\hline & & & White fat & 24 & 5.35 & 2.28 \\
\hline & & \multirow{12}{*}{ GSE54651* } & Adrenal gland & 8 & 5.29 & 5.40 \\
\hline & & & Aorta & 8 & 3.97 & 5.23 \\
\hline & & & Brainstem & 8 & 2.21 & 4.16 \\
\hline & & & Brown fat & 8 & 4.15 & 5.94 \\
\hline & & & Cerebellum & 8 & 4.06 & 4.86 \\
\hline & & & Heart & 8 & 4.73 & 6.23 \\
\hline & & & Hypothalamus & 8 & 2.19 & 4.19 \\
\hline & & & Kidney & 8 & 5.11 & 6.44 \\
\hline & & & Liver & 8 & 4.40 & 6.16 \\
\hline & & & Lung & 8 & 4.57 & 5.41 \\
\hline & & & Muscle & 8 & 5.26 & 5.24 \\
\hline & & & White fat & 8 & 3.71 & 3.87 \\
\hline
\end{tabular}

\footnotetext{
* denotes RNA-Seq data and others are microarray data.
} 

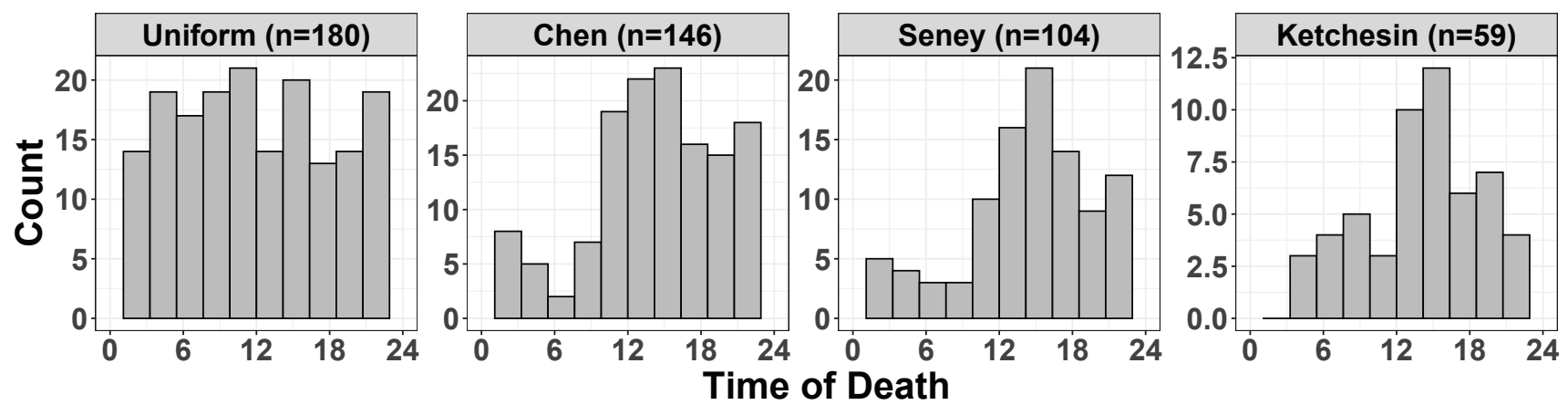

r=A/sigma $\square 0.4 \square 0.6 \square 0.8 \square 1$
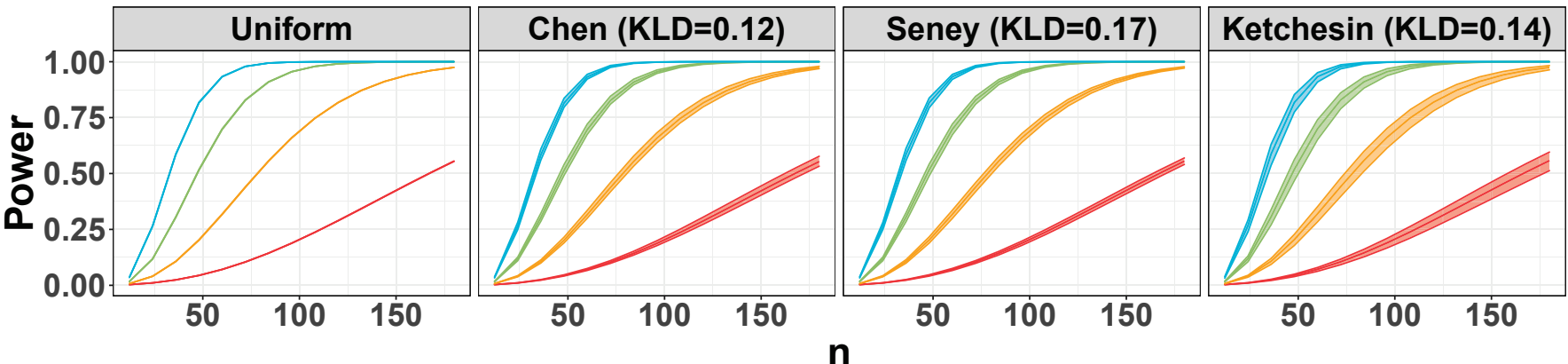

(a)

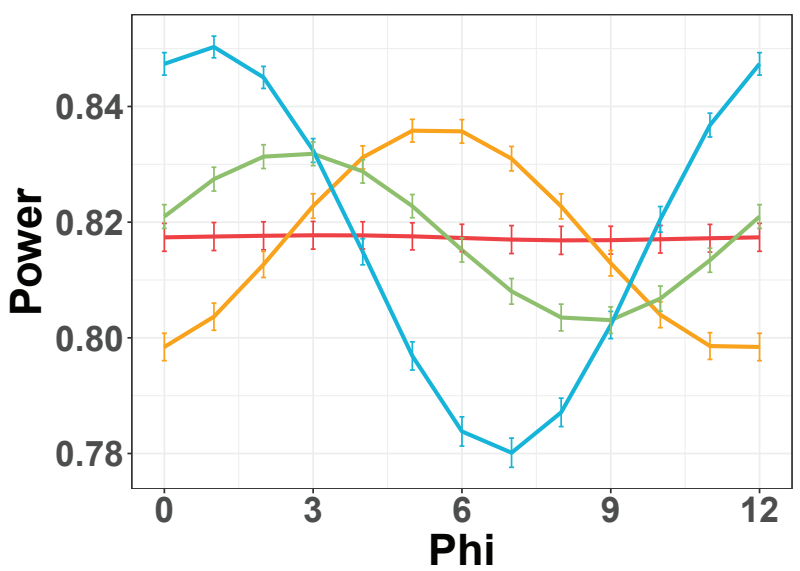

\section{Sampling design \\ - Uniform \\ - Chen \\ - Seney \\ - Ketchesin}

(b)

Figure 4. (a) demonstrates circadian power calculation using publicly available human datasets. The top panel shows the time of death distribution for Chen, Seney, and Ketchesin. The bottom panel shows the mean power trajectories of different study designs over 1000 repetitions with different intrinsic effect sizes $r=(0.4,0.6,0.8,1)$. The confidence bands represent the range of power achieved across phase values at $\phi=0,1,2,3, \ldots, 12$ for each scenario. (b) shows mean power trajectories across different $\phi$ when $n=120$ and $r=0.6$. For each $\phi$ and sampling distribution, we draw sampling times 1000 times and calculate corresponding power. Vertical bars indicate the $95 \%$ confidence interval of power estimates calculated form $\bar{x} \pm 1.96 s / \sqrt{1000}$ where $\bar{x}$ and $s$ are mean and standard deviation of power estimates respectively. Maximum power drop (calculated by $\frac{\text { max }_{\phi}\left(\text { mean_Power }_{-}-\text {min }_{\phi}\left(\text { mean_Power }_{-}\right)\right.}{\text {max }_{\phi}\left(\text { mean_Power }_{-}\right)}$is $4.8 \%, 3.5 \%$ and $8.3 \%$ respectively.

Our method has several advantages. To begin with, the theoretical framework suggests that the power calculation is related to the total effect size, which can be decomposed into sample size, intrinsic effect size (representing goodness-offit of circadian curve), and sampling design factor. Moreover, the sampling design factor brings about the concept of active design and passive design when samples are collected. This is an important concept in circadian experiment design, since the
ZT collection for human (passive design) and animal (active design) could be quite different. After that, we demonstrate the phase-invariant property of the evenly-spaced sampling design, which provides theoretical foundation for the design of many published circadian studies. In addition, the closedform formula in CircaPower allows unique inverse calculation of sample size given desired power at fast computing speed compared with the conventional MC approach. In this paper, 

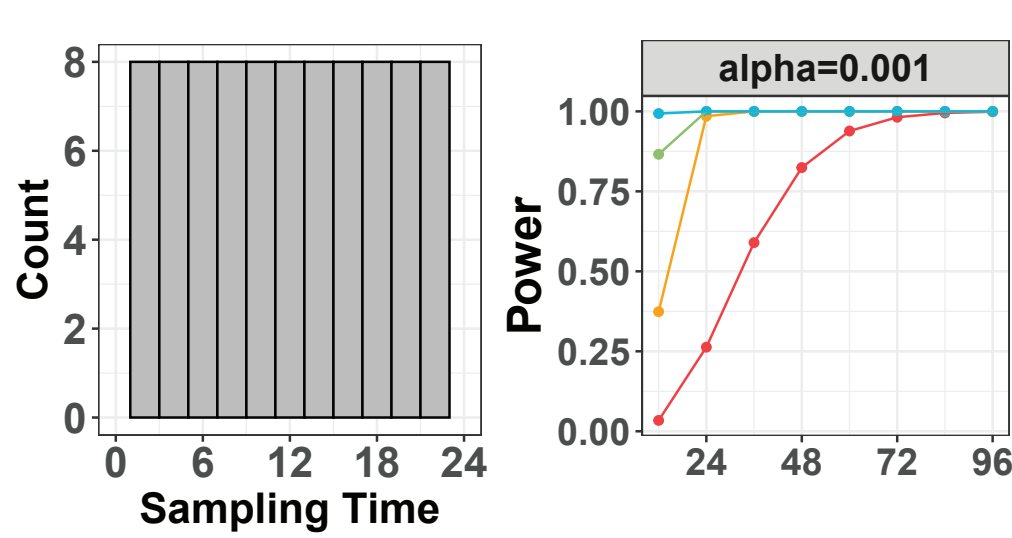

$\mathrm{r}=\mathrm{A} /$ sigma $\rightarrow 1 \rightarrow 2 \rightarrow 3 \rightarrow 4$

Figure 5. Circadian power calculation using publicly available mouse datasets. The left panel shows the distribution of one-sample one-period evenly-spaced design. The right panel shows the power trajectories for each of the type I error control $\alpha=(0.001,0.01,0.05)$ assuming intrinsic effect sizes $r=(1,2,3,4)$.

we also systematically examine the intrinsic effect sizes of published mouse or human gene expression circadian data, which could provide guidance for future researchers to design their transcriptomic circadian experiment when pilot data are not available. Although we present our work using transcriptomic data, CircaPower is applicable to other omics data, such as DNA methylation, ChIP-Seq proteomics, metabolomics, and clinical data (e.g., body temperature).

Our work has the following limitations and future work. Firstly, the current framework finds detection power by assuming the alpha level for type I error control. We intentionally select a more stringent alpha (e.g., alpha=0.001) to account for multiple comparison when thousands of genes are tested. Additional modeling is needed to extend for valid false discovery rate (FDR) control. Secondly, in addition to detecting genes with rhythmic pattern, another important research question is to identify differential circadian pattern $(10,35,40,46)$ (i.e., the circadian pattern is disrupted because of the treatment or condition). Extending the CircaPower framework to include power calculation for differential circadian analysis will be another future direction. Lastly, our work is based on a single cosinor model for circadian rhythmicity detection. While the cosinor model is advantageous statistically and show better type I error control when circadian assumption holds (10), extending the current framework to a more flexible family of circadian pattern is of biological interests to the general circadian research field.

To allow easy application by other researchers, our methods have been implemented in the $\mathrm{R}$ package CircaPower, which is publicly available in github (shttps://github.com/circaPower/circaPower).

\section{DATA AVAILABILITY}

Seney (39) is available through the Common Mind Consortium through an approval process (https://www.nimhgenetics.org/available_data/commonmind/). All other datasets are publicly available on the NCBI GEO database with accession no. shown in Table 1.

\section{ACKNOWLEDGEMENTS}

WZ and GT are funded by NIH grant R21LM012752. WZ, GT and CM are funded by NIH R01MH111601 and P50DA046346. KE and ZH are funded by R01HL153042. AL, $\mathrm{KE}$ and $\mathrm{ZH}$ are funded by R01AR079220.

Conflict of interest statement. None declared. 


\section{REFERENCES}

1. Lorena Aguilar-Arnal, Ofir Hakim, Vishal R Patel, Pierre Baldi, Gordon L Hager, and Paolo Sassone-Corsi. Cycles in spatial and temporal chromosomal organization driven by the circadian clock. Nature structural \& molecular biology, 20(10):1206-1213, 2013.

2. Pietro Badia, Brent Myers, Maren Boecker, Janice Culpepper, and JR Harsh. Bright light effects on body temperature, alertness, eeg and behavior. Physiology \& behavior, 50(3):583-588, 1991.

3. Molly S Bray, Chad A Shaw, Michael WS Moore, Rodrigo AP Garcia, Melissa M Zanquetta, David J Durgan, William J Jeong, Ju-Yun Tsai, Heiko Bugger, Dongfang Zhang, et al. Disruption of the circadian clock within the cardiomyocyte influences myocardial contractile function, metabolism, and gene expression. American Journal of Physiology-Heart and Circulatory Physiology, 294(2):H1036-H1047, 2008.

4. Angelo Cagnacci, JA Elliott, and SS Yen. Melatonin: a major regulator of the circadian rhythm of core temperature in humans. The Journal of Clinical Endocrinology \& Metabolism, 75(2):447-452, 1992.

5. Cho-Yi Chen, Ryan W Logan, Tianzhou Ma, David A Lewis, George C Tseng, Etienne Sibille, and Colleen A McClung. Effects of aging on circadian patterns of gene expression in the human prefrontal cortex. Proceedings of the National Academy of Sciences, 113(1):206-211, 2016

6. Han Cho, Xuan Zhao, Megumi Hatori, T Yu Ruth, Grant D Barish, Michael T Lam, Ling-Wa Chong, Luciano DiTacchio, Annette R Atkins, Christopher K Glass, et al. Regulation of circadian behaviour and metabolism by rev-erb- $\alpha$ and rev-erb- $\beta$. Nature, 485(7396):123-127, 2012

7. Germaine Cornelissen. Cosinor-based rhythmometry. Theoretical Biology and Medical Modelling, 11(1):16, 2014

8. Robert Dallmann, Antoine U Viola, Leila Tarokh, Christian Cajochen, and Steven A Brown. The human circadian metabolome. Proceedings of the National Academy of Sciences, 109(7):2625-2629, 2012.

9. DERK-JAN DIJK, Jeanne F Duffy, and Charles A Czeisler. Circadian and sleep/wake dependent aspects of subjective alertness and cognitive performance. Journal of sleep research, 1(2):112-117, 1992.

10. Haocheng Ding, Lingsong Meng, Andrew C Liu, Michelle L Gumz, Andrew J Bryant, Colleen A Mcclung, George C Tseng, Karyn A Esser, and Zhiguang Huo. Likelihood-based tests for detecting circadian rhythmicity and differential circadian patterns in transcriptomic applications. Briefings in Bioinformatics.

11. Jason R Gerstner, John N Koberstein, Adam J Watson, Nikolai Zapero, Davide Risso, Terence P Speed, Marcos G Frank, and Lucia Peixoto. Removal of unwanted variation reveals novel patterns of gene expression linked to sleep homeostasis in murine cortex. BMC genomics, 17(8):377_ 387,2016

12. Earl F Glynn, Jie Chen, and Arcady R Mushegian. Detecting periodic patterns in unevenly spaced gene expression time series using lombscargle periodograms. Bioinformatics, 22(3):310-316, 2006.

13. Brian A Hodge, Yuan Wen, Lance A Riley, Xiping Zhang, Jonathan $H$ England, Brianna D Harfmann, Elizabeth A Schroder, and Karyn A Esser. The endogenous molecular clock orchestrates the temporal separation of substrate metabolism in skeletal muscle. Skeletal muscle, 5(1):17, 2015.

14. Willemijntje A Hoogerwerf, Mala Sinha, Ana Conesa, Bruce A Luxon, Vahakn B Shahinian, Germaine Cornélissen, Franz Halberg, Jonathon Bostwick, John Timm, and Vincent M Cassone. Transcriptional profiling of mrna expression in the mouse distal colon. Gastroenterology, 135(6):2019-2029, 2008

15. M Hughes, L Deharo, SR Pulivarthy, J Gu, K Hayes, S Panda, and JB Hogenesch. High-resolution time course analysis of gene expression from pituitary. In Cold Spring Harbor Symposia on Ouantitative Biology, volume 72, pages 381-386. Cold Spring Harbor Laboratory Press, 2007.

16. Michael E Hughes, Katherine C Abruzzi, Ravi Allada, Ron Anafi, Alaaddin Bulak Arpat, Gad Asher, Pierre Baldi, Charissa De Bekker, Deborah Bell-Pedersen, Justin Blau, et al. Guidelines for genomescale analysis of biological rhythms. Journal of biological rhythms, 32(5):380-393, 2017.

17. Michael E Hughes, Luciano DiTacchio, Kevin R Hayes, Christopher Vollmers, S Pulivarthy, Julie E Baggs, Satchidananda Panda, and John B Hogenesch. Harmonics of circadian gene transcription in mammals. PLoS Genet, 5(4):e1000442, 2009

18. Michael E Hughes, John B Hogenesch, and Karl Kornacker. Jtk_cycle: an efficient nonparametric algorithm for detecting rhythmic components in genome-scale data sets. Journal of biological rhythms, 25(5):372-380, 2010
19. Christopher M Jung, Sat Bir S Khalsa, Frank AJL Scheer, Christian Cajochen, Steven W Lockley, Charles A Czeisler, and Kenneth P Wright Jr. Acute effects of bright light exposure on cortisol levels. Journal of biological rhythms, 25(3):208-216, 2010.

20. Kyle D Ketchesin, Wei Zong, Mariah A Hildebrand, Marianne L Seney, Kelly M Cahill, Madeline R Scott, Vaishnavi G Shankar, Jill R Glausier, David A Lewis, George C Tseng, et al. Diurnal rhythms across the human dorsal and ventral striatum. Paroceedings of the National Academy of Sciences, 118(2), 2021

21. Nobuya Koike, Seung-Hee Yoo, Hung-Chung Huang, Vivek Kumar, Choogon Lee, Tae-Kyung Kim, and Joseph S Takahashi. Transcriptional architecture and chromatin landscape of the core circadian clock in mammals. science, 338(6105):349-354, 2012

22. Jiajia Li, Gregory R Grant, John B Hogenesch, and Michael E Hughes. Considerations for rna-seq analysis of circadian rhythms. Methods in enzymology, 551:349-367, 2015.

23. Jun Z Li, Blynn G Bunney, Fan Meng, Megan H Hagenauer, David M Walsh, Marquis P Vawter, Simon J Evans, Prabhakara V Choudary, Preston Cartagena, Jack D Barchas, et al. Circadian patterns of gene expression in the human brain and disruption in major depressive disorder. Proceedings of the National Academy of Sciences, 110(24):9950-9955, 2013.

24. Andrew SP Lim, Hans-Ulrich Klein, Lei Yu, Lori B Chibnik, Sanam Ali, Jishu Xu, David A Bennett, and Philip L De Jager. Diurnal and seasonal molecular rhythms in human neocortex and their relation to alzheimer's disease. Nature communications, 8(1):1-16, 2017.

25. Andrew SP Lim, Gyan P Srivastava, Lei Yu, Lori B Chibnik, Jishu $\mathrm{Xu}$, Aron S Buchman, Julie A Schneider, Amanda J Myers, David A Bennett, and Philip L De Jager. 24-hour rhythms of dna methylation and their relation with rhythms of rna expression in the human dorsolateral prefrontal cortex. PLoS genetics, 10(11):e1004792, 2014.

26. Leonidas S Lundell, Evelyn B Parr, Brooke L Devlin, Lars R Ingerslev, Ali Altıntaş, Shogo Sato, Paolo Sassone-Corsi, Romain Barrès, Juleen R Zierath, and John A Hawley. Time-restricted feeding alters lipid and amino acid metabolite rhythmicity without perturbing clock gene expression. Nature communications, 11(1):1-11, 2020.

27. Danyi Ma, Meng Zhao, Lucheng Xie, Qingqin Wu, Lingfeng Gou, Chuanzhen Zhu, Yuqi Fan, Haifang Wang, Jun Yan, et al. Spatiotemporal single-cell analysis of gene expression in the mouse suprachiasmatic nucleus. Nature neuroscience, 23(3):456-467, 2020.

28. Murakami Mari, Paola Tognini, Yu Liu, L Kristin, Pierre Baldi, and Sassone-Corsi Paolo. Gut microbiota directs ppargamma-driven reprogramming of the liver circadian clock by nutritional challenge. 2016.

29. Selma Masri, Thales Papagiannakopoulos, Kenichiro Kinouchi, Yu Liu, Marlene Cervantes, Pierre Baldi, Tyler Jacks, and Paolo Sassone-Corsi. Lung adenocarcinoma distally rewires hepatic circadian homeostasis. Cell, 165(4):896-909, 2016.

30. Selma Masri, Paul Rigor, Marlene Cervantes, Nicholas Ceglia, Carlos Sebastian, Cuiying Xiao, Manuel Roqueta-Rivera, Chuxia Deng, Timothy F Osborne, Raul Mostoslavsky, et al. Partitioning circadian transcription by sirt6 leads to segregated control of cellular metabolism. Cell, 158(3):659-672, 2014.

31. Carla S Möller-Levet, Simon N Archer, Giselda Bucca, Emma E Laing, Ana Slak, Renata Kabiljo, June CY Lo, Nayantara Santhi, Malcolm von Schantz, Colin P Smith, et al. Effects of insufficient sleep on circadian rhythmicity and expression amplitude of the human blood transcriptome. Proceedings of the National Academy of Sciences, 110(12):E1132-E1141, 2013

32. John F Monahan. A primer on linear models. CRC Press, 2008.

33. Young-Ji Na, Jung Hwan Sung, Suk Chan Lee, Young-Ju Lee, Yeun Joo Choi, Woong-Yang Park, Hee Sup Shin, and Ju Han Kim. Comprehensive analysis of microrna-mrna co-expression in circadian rhythm. Experimental \& molecular medicine, 41(9):638-647, 2009.

34. Svetlana Nikolaeva, Sylvain Pradervand, Gabriel Centeno, Vlasta Zavadova, Natsuko Tokonami, Marc Maillard, Olivier Bonny, and Dmitri Firsov. The circadian clock modulates renal sodium handling. Journal of the American Society of Nephrology, 23(6):1019-1026, 2012.

35. Rex Parsons, Richard Parsons, Nicholas Garner, Henrik Oster, and Oliver Rawashdeh. Circacompare: a method to estimate and statistically support differences in mesor, amplitude and phase, between circadian rhythms. Bioinformatics, 36(4):1208-1212, 2020.

36. Georgios K Paschos, Salam Ibrahim, Wen-Liang Song, Takeshige Kunieda, Gregory Grant, Teresa M Reyes, Christopher A Bradfield, Cheryl H Vaughan, Michael Eiden, Mojgan Masoodi, et al. Obesity in 
mice with adipocyte-specific deletion of clock component arntl. Nature medicine, 18(12):1768-1777, 2012.

37. Marc D Ruben, Gang Wu, David F Smith, Robert E Schmidt, Lauren J Francey, Yin Yeng Lee, Ron C Anafi, and John B Hogenesch. A database of tissue-specific rhythmically expressed human genes has potential applications in circadian medicine. Science Translational Medicine, 10(458), 2018

38. Shogo Sato, Astrid Linde Basse, Milena Schönke, Siwei Chen, Muntaha Samad, Ali Altıntass, Rhianna C Laker, Emilie Dalbram, Romain Barrès, Pierre Baldi, et al. Time of exercise specifies the impact on muscle metabolic pathways and systemic energy homeostasis. Cell metabolism, 30(1):92-110, 2019.

39. Marianne L Seney, Kelly Cahill, John F Enwright, Ryan W Logan, Zhiguang Huo, Wei Zong, George Tseng, and Colleen A McClung. Diurnal rhythms in gene expression in the prefrontal cortex in schizophrenia. Nature communications, 10(1):1-11, 2019.

40. Jordan M Singer and Jacob J Hughey. Limorhyde: a flexible approach for differential analysis of rhythmic transcriptome data. Journal of biological rhythms, 34(1):5-18, 2019.

41. Guiomar Solanas, Francisca Oliveira Peixoto, Eusebio Perdiguero, Mercè Jardí, Vanessa Ruiz-Bonilla, Debayan Datta, Aikaterini Symeonidi, Andrés Castellanos, Patrick-Simon Welz, Juan Martín Caballero, et al. Aged stem cells reprogram their daily rhythmic functions to adapt to stress. Cell, 170(4):678-692, 2017.

42. Dirk Jan Stenvers, Aldo Jongejan, Sadaf Atiqi, Jeroen P Vreijling, Eelkje J Limonard, Erik Endert, Frank Baas, Perry D Moerland, Eric Fliers, Andries Kalsbeek, et al. Diurnal rhythms in the white adipose tissue transcriptome are disturbed in obese individuals with type 2 diabetes compared with lean control individuals. Diabetologia, 62(4):704-716, 2019.

43. Martin Straume. Dna microarray time series analysis: automated statistical assessment of circadian rhythms in gene expression patterning. In Methods in enzymology, volume 383, pages 149-166. Elsevier, 2004.

44. Joseph S Takahashi. Transcriptional architecture of the mammalian circadian clock. Nature Reviews Genetics, 18(3):164, 2017.

45. Paul F Thaben and Pål O Westermark. Detecting rhythms in time series with rain. Journal of biological rhythms, 29(6):391-400, 2014

46. Paul F Thaben and Pål O Westermark. Differential rhythmicity: detecting altered rhythmicity in biological data. Bioinformatics, 32(18):2800-2808, 2016.

47. Yunzhi Wang, Lei Song, Mingwei Liu, Rui Ge, Quan Zhou, Wanlin Liu, Ruiyang Li, Jingbo Qie, Bei Zhen, Yi Wang, et al. A proteomics landscape of circadian clock in mouse liver. Nature communications, 9(1):1-16, 2018.

48. Rendong Yang and Zhen Su. Analyzing circadian expression data by harmonic regression based on autoregressive spectral estimation. Bioinformatics, 26(12):i168-i174, 2010.

49. Ray Zhang, Nicholas F Lahens, Heather I Ballance, Michael E Hughes, and John B Hogenesch. A circadian gene expression atlas in mammals: implications for biology and medicine. Proceedings of the National Academy of Sciences, 111(45):16219-16224, 2014. 